\title{
Molecular Epidemiology of HIV-1 in Venezuela: High Prevalence of HIV-1 Subtype B and Identification of a B/F Recombinant Infection
}

\author{
$* †$ Erika Castro, ‡Gloria Echeverría, $\neq$ Leopoldo Deibis, §Beatríz González de Salmen, \\ *Aline Dos Santos Moreira, *Monick L. Guimarães, "Francisco I. Bastos, and \\ *Mariza G. Morgado
}

\begin{abstract}
*Department of Immunology, Oswaldo Cruz Institute, FIOCRUZ, Rio de Janeiro, Brazil; †Non Governmental Organization, CRIATEII, Porlamar, Margarita Island, Venezuela; \$Immunology Institute, Central University of Venezuela, Caracas, Venezuela; $\S S T D$ s and AIDS Program, State Hospital "Luis Ortega," Porlamar, Margarita Island, Venezuela; and "Department of Health Information, CICT, FIOCRUZ, Rio de Janeiro, Brazil
\end{abstract}

This study was supported by grants of the National Council for Scientific and Technological Research from Venezuela "CONICIT," PAHO/Venezuela, and the Oswaldo Cruz Institute.

Results shown in this report are part of the $\mathrm{PhD}$ thesis of Erika Castro in the field of Tropical Medicine from the Oswaldo Cruz Institute, FIOCRUZ, Rio de Janeiro, Brazil. Current address for Erika Castro: Instituto de Inmunología, Universidad Central de Venezuela, Ciudad Universitaria, Los Chaguaramos, Caracas, 1050-A, Venezuela; e-mail:ebatenjer@hotmail.com

Address correspondence and reprint requests to Mariza G. Morgado, Laboratory of AIDS \& Molecular Immunology, Dept. of Immunology, Oswaldo Cruz Institute/Fiocruz-UNAIDS Collaborating Centre, Avenida Brasil 4365, Manguinhos, Rio de Janeiro, CEP 21045-900, RJ, Brazil; e-mail: mmorgado@ioc.fiocruz.br

Manuscript received February 5, 2002; accepted October 21, 2002.
Since the beginning of the AIDS epidemic, HIV-1 polymorphism has been an important issue for HIV/AIDS researchers worldwide. As a consequence of this effort to monitor HIV-1 diversity, 24 major genetic forms, including 9 distinct subtypes (A-D, F, G, H, J, and $\mathrm{K}$ ) and 14 circulating recombinant forms (CRFs; CRF01_AE, CRF02_AG, CRF03_AB, CRF04_cpx, CRF05_DF, CRF06_cpx, CRF07_BC, CRF08_BC, CRF09_?, CRF10_CD, CRF11_cpx, CRF12_BF, CRF13_cpx, and CRF14_BG), have already been reported for the major group (1-3).

Data from South American countries are also included in such studies, showing predominantly the presence of 
subtype B. Briefly, subtypes B, C, D, variant $\mathrm{B}^{\prime \prime}$ from subtype $\mathrm{B}$, and the recombinant $\mathrm{B} / \mathrm{F}$ and $\mathrm{B} / \mathrm{C}$ forms have been identified in Brazil (4-13). Reports from other countries of the region have shown the existence of subtypes B, C, F, and the recombinant B/F form in Argentina $(14,15)$; subtypes B and E in Uruguay $(16,17)$; subtypes B and F in Bolivia (18); and subtype B in Colombia (19), Paraguay (20), Honduras (21), and Mexico (22). In addition to subtype B in Chile, a single case of subtype A infection has been reported (23). Venezuela's earliest research on HIV-1 polymorphism verified the presence of subtype $B$ in a small number of samples $(24,25)$ and, more recently, the occurrence of an F/B protease/reverse transcriptase recombinant strain in 1 person participating in an UNAIDS study (26).

We conducted a survey to analyze HIV-1 variability and epidemiologic characteristics of infected persons from Caracas and Margarita Island, the two localities with the highest morbidity rates for AIDS in Venezuela (27). This study also represents the first contribution toward the establishment of the Venezuelan National Program on HIV-1 Surveillance of Molecular Variability.

\section{METHODS}

\section{Patients and Methods}

The recruitment of patients and the collection of biologic sample were carried out from June 1998 to October 1999. Two independent convenience samples of outpatients were enrolled in the study: one from Margarita Island (STDs Department of the State Hospital), and a second from Caracas (Retrovirus Reference Center of the Institute of Immunology). Only confirmed HIV-1-infected (by Western blot or immunofluorescence) patients were included in the study.

This study included 72 HIV-1-infected patients, 22 from Margarita Island and 50 from Caracas. The Caracas sample was a pool of persons living in 12 of the 23 states of the country seeking specialized care in the reference center, located in the capital. All volunteers answered a questionnaire addressing sociodemographic and behavioral data, signed informed consent, and allowed the peripheral extraction of $5 \mathrm{~mL}$ of whole blood.

\section{HIV-1 Subtype Characterization}

Five milliliters of ethylenediamine tetraacetic (EDTA) anticoagulated blood were obtained from each patient. The plasma was collected, and the remaining white cell layer $(2-3 \mathrm{~mL})$ was resuspended in $0.5 \%$ Saponin $/ 0.4 \% \mathrm{NaCl}$ (vol/vol), thoroughly vortexed, centrifuged $(400 \mathrm{~g}$ for 5 minutes at room temperature), and washed twice with phosphatebuffered saline (PBS) by centrifugation in the same conditions. Resulting pellets were stored at $-20^{\circ} \mathrm{C}$ until proviral DNA extraction at FIOCRUZ, Brazil. Proviral DNA was extracted by the proteinase K-SDS/phenol/chloroform method as previously described (4).

A nested polymerase chain reaction (PCR) protocol was used to amplify the gp120 C2-C3 (ED31-ED33 primer set) using ED3-ED14 as outer primers $(28,29)$. DNA samples that did not give amplified products using this protocol were amplified with other primer sets like ED5-ED12, ES7-ES8, or other combinations between them, according to the algorithm shown in Figure 1. PCR-negative samples for the env region primers were further analyzed with a multiplex PCR protocol (30), with modified env primers (ED5-ED12 [outer]/ED31-ED33 [inner] primers, in substitution of those described in the original reference), to check the positivity to HIV genome.

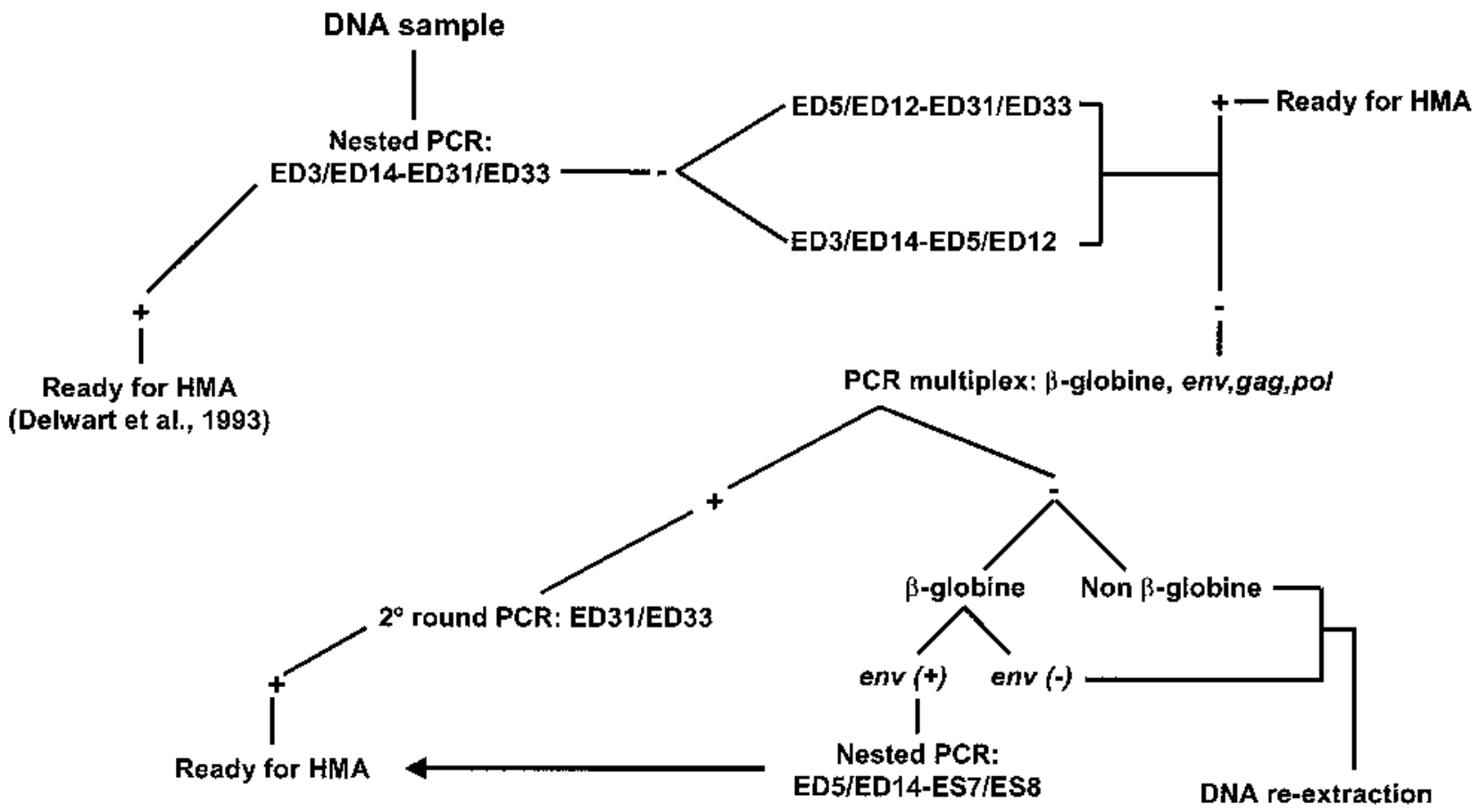

FIG. 1. PCR algorithm used on HIV-1 proviral DNA samples from Venezuela, 1998 to 1999. 
HIV-1 subtyping was determined by env heteroduplex mobility assay (HMA) $(28,29,31)$ using PCR amplified reference plasmids of HIV-1 subtypes B-F, or A-H in doubtful cases. The NIH AIDS Research and Reference Reagent Program provided the HMA HIV-1 env subtyping kit. In order to identify B" GWGR subtype B samples, $5 \mu \mathrm{L}$ of ED31/ED33 PCR products were further digested with $6 \mathrm{U}$ of Fok I restriction enzyme (11).

\section{Sequencing of PCR Products}

A sample of $5 \mu \mathrm{L}$ of the C2-V3 ED31/ED33 PCR reaction was submitted to enzymatic treatment with $1 \mu \mathrm{L}$ of shrimp alkaline phosphatase $(10 \mathrm{U} / \mu \mathrm{L})$ and $1 \mu \mathrm{L}$ of exonuclease I $(2 \mathrm{U} / \mu \mathrm{L})$ hydrolytic enzymes according to the manufacturer's protocol and directly sequenced using the dideoxy chain termination method kit (DNA sequencer model 377, Applied Biosystems, Foster City, CA, U.S.A.), using the Big Dye Terminator kit (Applied Biosystems, Foster City, CA, U.S.A.). The PCR products were sequenced in both senses with ED31, ED33, and ES7 oligonucleotides as sequencing primers. Alignment of multiple nucleotide and predicted amino acid sequences was performed with the University of Wisconsin Genetic Computer Group (GCG) package. The subtype F env sample identified in the current study was further sequenced in the protease and reverse transcriptase (RT) regions using, respectively, DP10/DP11 (outer)/DP16/DP17 (inner) protease primer sets (12) and RT9/RT12 (outer)/RT1/RT4 (inner) RT primer sets (32) to complement its analyses.

Phylogenetic analysis was done using the CLUSTAL W software (33). Phylogenetic tree constructions were performed using the neighbor-joining method, and the reliability of the branching orders was determined by the 100 times bootstrap method. The final trees were visualized using the Tree View program (34). The intrasubtype distances were calculated using the Kimura two-parameter with pairwise gap deletion based on the MEGA program (35). GenBank accession numbers for HIV-1 env sequences from Margarita Island and Caracas, Venezuela are assigned from AF467716 to AF467725 (Accession numbers for $99 \mathrm{VenC} 13$ protease and $\mathrm{RT}$ sequences are, respectively, AF467727 and AF 467726.).

\section{Statistical Analysis}

The $\chi^{2}$ or Fisher exact test was employed to evaluate possible associations, and a $p$ level of $<.05$ was defined as statistically significant.

\section{RESULTS}

Caracas and Margarita Island samples were substantially different in sociodemographic information. The Caracas sample $(n=50)$ was composed basically of men (46 men, 4 women), while the Margarita Island sample $(n=22)$ had exactly the same number of men and women. Patients from Margarita Island were younger (half were aged 18-30 years), contrasting with a concentration (64\%) of patients from Caracas aged 31 to 44 years. Roughly $73 \%$ of the Margarita Island patients were supposed to be infected by heterosexual transmission, while the vast majority (70\%) of patients from Caracas were gay men. CD4 counts were roughly com- parable in patients from these two cities (data not shown).

\section{DNA Amplification and HIV Subtyping}

With the primer sets ED3/14-ED31/33 we could amplify $60 \%$ DNA products from the Margarita Island samples and $82 \%$ of the Caracas samples, respectively. The remaining DNA products were analyzed with other primer sets according to the algorithm presented in Figure 1. There was no correlation between primer sets ED3/14-ED31/33 amplification capacity with either exposure category or $\mathrm{CD}^{+}$counts in the two samples. A significantly $(p=.038)$ higher discrimination capacity of primer sets ED3/14-ED31/33 was found for the Caracas samples, if compared with the Margarita Island samples, pointing to a higher intrasubtype B variability in the samples of Margarita Island vis-à-vis the samples from the continent, possibly due to the close link of the Island with the Caribbean subepidemic.

Three cases (99VenM1, 99VenC17, and 99VenC30) failed all PCR attempts of amplification. We repeated the blood screening with new samples obtained from 2 of these patients (99VenM1 and 99VenC17), and again we failed to amplify proviral DNA. Viral load (Nuclisens NASBA Diagnostics HIV-1 RNA QT) in these patients were 700 copies $/ \mathrm{mL}$ and 14,000 copies $/ \mathrm{mL}$ for 99VenM1 and 99VenC17, respectively, and their $\mathrm{CD}^{+}$ counts were $>350$ cells $/ \mathrm{mL}$. Both patients remained asymptomatic during the period they were followed (1998-1999) and did not receive any kind of antiretroviral drugs in this period. As PCR sensitivity in our study could successfully amplify from $>20$ copies of proviral DNA, we tried alternative sources of genomic material, obtaining cDNA from the plasmatic RNA retrotranscription. In these 2 cases, amplification was carried out with a multiplex PCR directed to viral env, gag, and pol genes, and a positive band for gag gene was observed only for the sample of patient 99VenC17 (data not shown).

We identified the subtype B HMA profile of HIV-1 in $20(90.9 \%)$ of the Margarita Island patients and in 47 (94.0\%) of the Caracas study group. One patient in the Caracas sample (99VenC13) showed the subtype F migration pattern. He was a 34-year-old gay man, with advanced immunosuppression $\left(\mathrm{CD}^{+}{ }^{+}\right.$counts of 122 cells $/ \mathrm{mm}^{3}$ ). He was presumably infected between 1994 and 1996, when traveling abroad. HMA result was indeterminate for one sample (99VenM11) from the Margarita sample, which was further selected for DNA sequencing. 
According to the FOK I RFLP, none of the subtype B virus described in this study exhibited the typical GWGR subtype B profile in the V3 loop.

A subset of 11 samples, including the indeterminate HIV-1 subtype from the Margarita Island group (99VenM11), the subtype F env sample (99VenC13) from the Caracas group, and 9 subtype B samples, was selected for the C2-V3 env sequencing (Fig. 2A). The phylogenetic tree confirms that sample $99 \mathrm{VenC} 13$ does cluster within the $\mathrm{F}$ clade in the $\mathrm{C} 2-\mathrm{V} 3$ env region with a high bootstrap value. CRF-12 B/F reference samples (36) from Argentina (ARMA 185 and ARMA 159) were included in this analysis and also clustered within the subtype F branches. However, sample V62, a B/F recombinant virus previously described from Venezuela (26), clustered with the subtype B references in the env $\mathrm{C} 2-\mathrm{V} 3$ region. The remaining samples from Caracas and Margarita Island clustered into the B subtype with a bootstrap value of $70 \%$, reflecting a higher interpatient diversity in this group. Indeed, no specific clustering could be detected for the HIV-1 samples from Venezuela, which were interspersed in branches of subtype B reference samples from other South American countries and from the United States. Moreover, pairwise distance among the subtype B specimens was, on average, $18.7 \%$ at the nucleotide level.

Additional phylogenetic analysis of env subtype $\mathrm{F}$ sample $99 \mathrm{VenC} 13$ in the protease and RT regions (Fig. $2 \mathrm{~B}, \mathrm{C})$ revealed a mosaic genome clustering with subtype $\mathrm{F}$ references in the protease region, whereas for the RT fragment, this sample clustered in a separated branch with the two CRF-12 B/F ARMA 185 and ARMA 159 references with high bootstrap value $(95 \%)$, indicating a recombinant profile in this fragment.

The predicted amino acid sequences of the env gp120 C2-V3 region obtained from Venezuelan HIV-1 strains are presented in Figure 3. Nine subtype B and one subtype $\mathrm{F}$ amino acid sequences were aligned with $\mathrm{B}$ and $\mathrm{F}$ reference sequences previously reported from other South American countries [Argentina (14), Bolivia (18), Brazil (13), and Uruguay (17)] and with one subtype F sequence from Romania (37). A broad spectrum of tetrameric amino acid sequences was observed at the crown of the V3 loop, including sequences containing the GPGRTW (99VenM11 and 99VenC28) and GPGGAF (99VenC47) motives for subtype B. The GPGR motif was also verified in the subtype $\mathrm{F}$ sequence from Venezuela, as described for other South American subtype F samples (37), distinguishing them from the Romanian subtype $\mathrm{F}$ viruses.

\section{DISCUSSION}

Based on env and pol (PR and RT) HMA and nucleotide sequence analyses, respectively, this study identifies the presence of a new HIV-1 B/F recombinant virus in Venezuela (isolate 99VenC13). This sample was obtained from a gay man with advanced immunosuppression who was presumably infected between 1994 and 1996 when traveling abroad. Another B/F recombinant virus was previously described in Venezuela (26), but in that case, the env and RT regions were subtyped as $\mathrm{B}$, while the PR was F, thus corresponding to an Fpro/Brt/Benv mosaic genome. In the current study, however, sample 99VenC13 clustered with subtype F reference samples in both env and PR regions and with the CRF_12 B/F references (ARMA 159 and ARMA 185 ) in the RT, corresponding to an $\mathrm{Fpro} / \mathrm{B} / \mathrm{Frt} / \mathrm{Fenv}$ recombinant genome. This particular observation could be linked to the increasing description of recombinant viruses and CRFs worldwide (3), including the recent description of CRF_12 B/F and other B/F recombinant viruses in some South American countries $(5,15,36,38)$. The relevant differences between continental and islander's samples suggest HIV-1 variability in Venezuela should be further explored. Recent studies carried out in Argentina (39) show a wide circulation of B/F recombinant strains among different exposure categories in Buenos Aires. The signature on the top of the V3 loop of 99VenC13 (GPGRVV), associated with its clustering with sub-subtype F1 reference samples in the phylogenetic analysis, allows us to consider this subtype $\mathrm{F}$ strain in the F1 subgroup (40), typically observed in South American subtype F strains $(4,9)$.

The presence of mosaic genomes highlights the need to improve subtyping protocols with the molecular analysis of at least two distinct viral regions to identify the circulation of recombinant forms and dual infection

FIG. 2. Phylogenetic analysis of Venezuelan HIV-1 subtypes. (A) C2-V3 env sequences were compared with reference sequences of the HIV-1 group M subtypes available in the Los Alamos database, HIV-1 B and F samples from South America countries $(13,14,17,18)$, and CRF_12 B/F representative samples (36). (B) Protease and (C) reverse transcriptase sequences of sample $99 \mathrm{VenC13}$ were respectively compared with reference sequences of the HIV-1 group $\mathrm{M}$ subtypes available in the Los Alamos database and CRF_12 B/F representative samples (36). Sequence CPZgab was used as outgroup. Aligned fragments of 420bp (C2-V3), 300bp (protease), and $500 \mathrm{bp}$ (reverse transcriptase) were analyzed as described in the text, and bootstrap values for 100 replicates are listed at the major subtype branches. Venezuelan samples sequenced in this study are italicized in bold. GenBank accession numbers are specified in the Material and Methods section. 


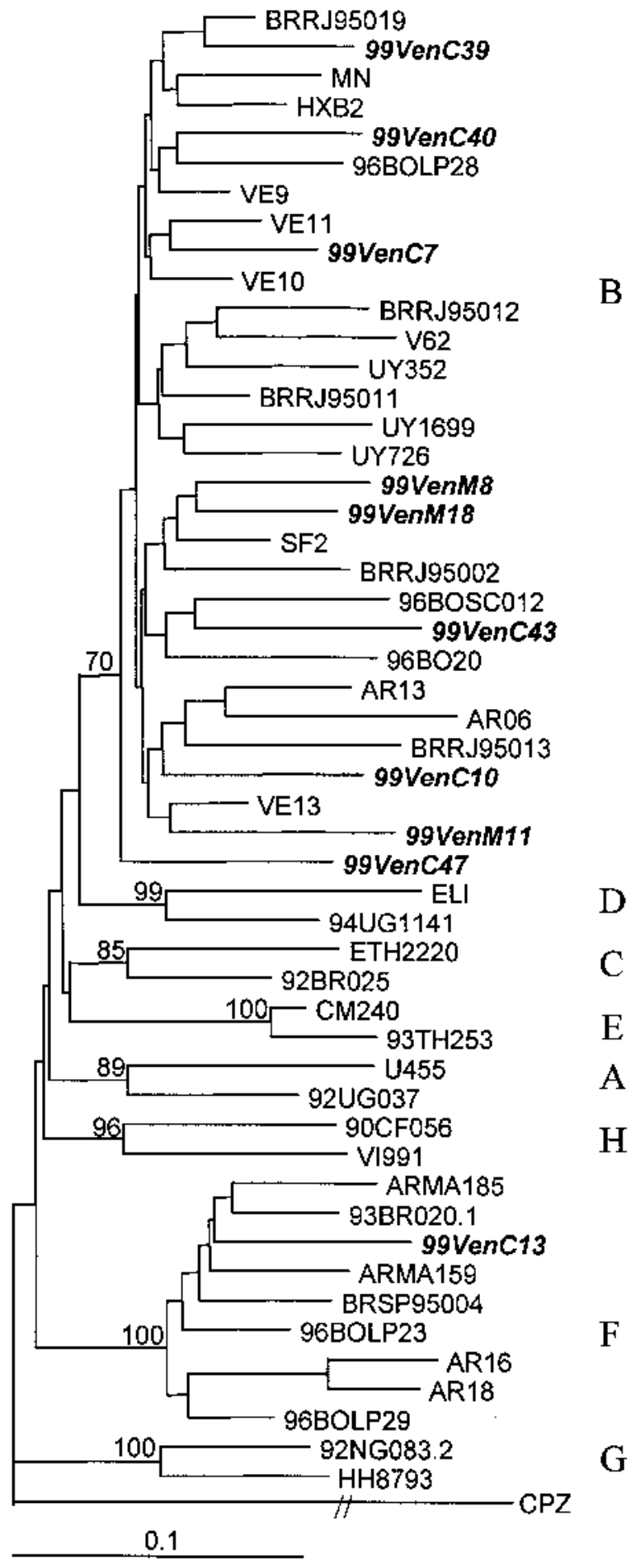

A

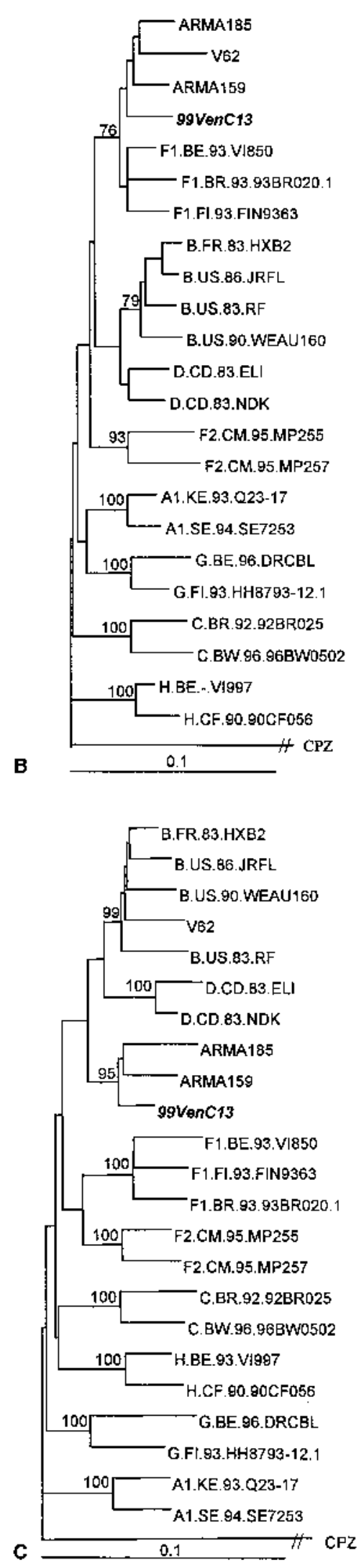




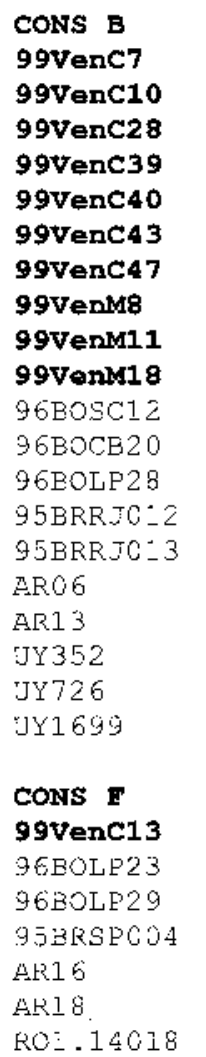

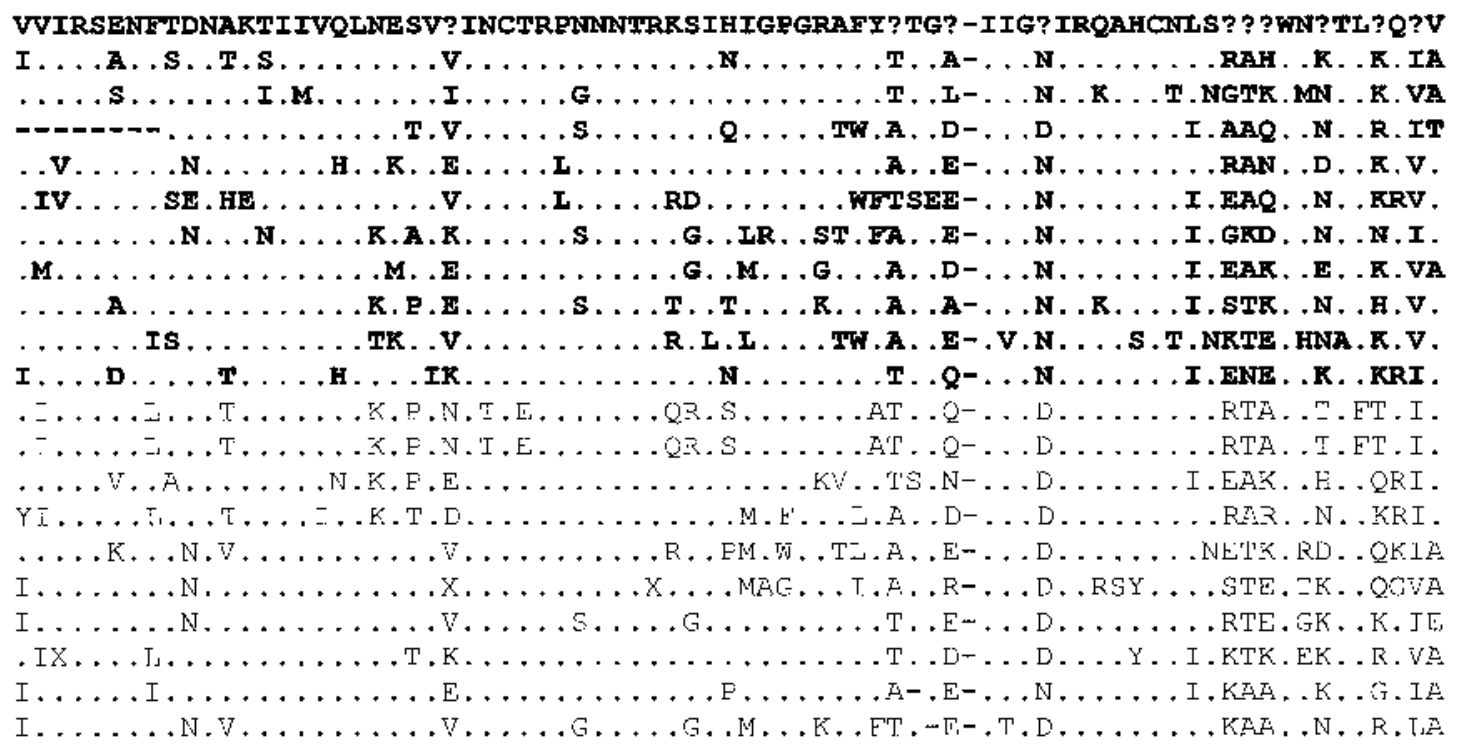

IIIRSQN I SDNAKTI IVHLNESVQINCTRPNAN ITRS I ? IGPGRAFY? TG? -I IGDIRKAHCNVSG? ?WN? TLE?V?

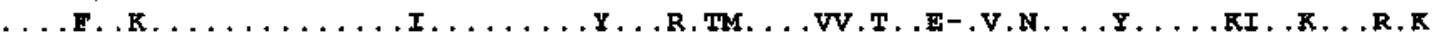

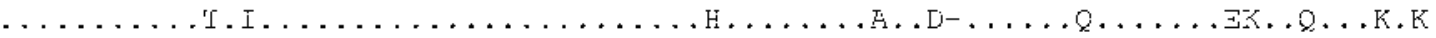
$\ldots \ldots \ldots \ldots$ I. . . . . . . . . . . . . . . .N, . . . . . . . . .

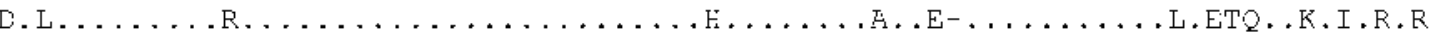

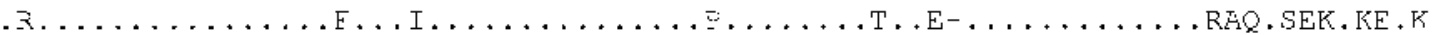

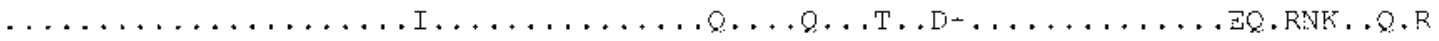

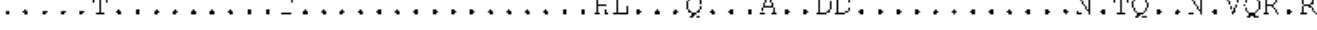

FIG. 3. Alignment of deduced $\mathrm{C} 2-\mathrm{V} 3$ env region amino acid sequences obtained from Venezuelan HIV-1 strains compared with the subtype B and F consensus sequences and individual reference samples from Argentina (14), Bolivia (18), Brazil (13), Uruguay (17), and Romania (37). Dots indicate identity with the consensus sequence, and dashes indicate gaps introduced to optimize the sequence alignment.

with different subtypes (41). For this purpose, an HMA env/gag protocol has been recently developed (42). In addition, today's effort on HIV pol genotyping as a tool to document HIV-1 strains resistant to antiretroviral drugs has lead to a valuable source of data for phylogenetic analysis and subtyping worldwide $(26,43)$.

In the B subtype samples, we found motifs GPGGAF and GPGRTW, which were never described before among Venezuelan HIV-1 B subtypes $(24,25)$, probably reflecting the evolving molecular epidemiology of HIV-1 in Venezuela. Nevertheless, motif GPGGAF (99VenM11 and 99VenC47) in the top of the V3 loop was previously described in subtype B strains circulating in Brazil (4). Deserving special attention is the finding of the signature GPGRTW (99VenC28), which, to the best of our knowledge, was never described before for subtype B strains circulating in Latin American countries. The Venezuelan B subtypes cluster within different geographical HIV-1 subtype B reference samples, pointing to an important diversity, especially considering the small number of B strains analyzed in this study. Alignment of the $\mathrm{C} 2-\mathrm{V} 3$ region with Venezuelan reference subtype B samples previously described $(24,25)$ was not possible because of the difference of the fragment region evaluated in such samples, which provided a very small alignment extension when tested.

The study highlights the pressing need to comprehensively monitor HIV-1 molecular epidemiology in a geographic region in which the HIV/AIDS subepidemic is still rapidly spreading and becoming more complex and interactive, combining South America continental characteristics with patterns closely related to the Caribbean subepidemics.

Acknowledgments: The authors thank Natalia Sandoval from the Anticancer Society Laboratory at Margarita Island for her important support. The authors also thank Hector Bárcenas ("AIDS for AIDS") for his invaluable help with the volunteers from Caracas.

\section{REFERENCES}

1. Korber B, Kuiken C, Foley B, et al. Human retroviruses and AIDS. Theoretical biology and biophysics. Los Alamos, NM: Los Alamos National Laboratory, 1998.

2. Robertson DL, Anderson JP, Bradac JA, et al. HIV-1 nomenclature proposal. Science 2000;288:55-6.

3. HIV Sequence Database. http://hiv-web.lanl.gov/CRFs/CRFs.html.

4. Morgado MG, Sabino ES, Shpaer EG, et al. V3 region polymorphism in HIV-1 from Brazil: prevalence of subtype B strains di- 
vergent from North American/European prototype and detection of subtype F. AIDS Res Hum Retroviruses 1994;10:569-76.

5. Sabino E, Shpaer EG, Morgado MG, et al. Identification of human immunodeficiency virus type 1 envelope genes recombinant between subtypes $\mathrm{B}$ and $\mathrm{F}$ in two epidemiologically linked individuals from Brazil. J Virol 1994;68:6340-6.

6. Da Costa S, Schechter M, Shindo N, et al. Sequence and phylogenetic analysis of glycoprotein 120 of an HIV type 1 variant (GWGR) prevalent in Brazil. AIDS Res Hum Retroviruses 1995;11:1243-5.

7. Pinto M, Tanuri A, Schechter M. Frequency of subtypes of HIV-1 in participants of a cohort study in Rio de Janeiro, Brazil, as suggested by endonuclease digestion patterns of the protease gene [abstract 1:133]. Presented at the XI International Conference on AIDS, Vancouver, 1996.

8. Sabino E, Diaz R, Brigido R, et al. Distribution of HIV-1 subtypes seen in an AIDS clinic in São Paulo City, Brazil. AIDS 1996;10: 1579-84.

9. Galvão-Castro B, Couto-Fernández JC, Mello MA, et al. The Brazilian network for the HIV-1 isolation and characterization. A nationwide effort to systematically monitor HIV-1 diversity in Brazil: preliminary results. Mem Inst Oswaldo Cruz 1996;91:335-8.

10. Morgado MG, Guimarães ML, Gripp CBG, et al. and The Hospital Evandro Chagas AIDS Clinical Research Group. Molecular epidemiology of HIV-1 in Brazil: high prevalence of HIV-1 subtype B and identification of an HIV-1 subtype D infection in the city of Rio de Janeiro, Brazil. J Acquir Immune Defic Syndr 1998;18:488-94.

11. Morgado MG, Guimarães ML, Neves I Jr, et al. and The Hospital Evandro Chagas AIDS Clinical Research Group. Molecular epidemiology of HIV in Brazil: polymorphism of the antigenically distinct HIV-1 B subtype strains. Mem Inst Oswaldo Cruz 1998;93:383-6.

12. Tanuri A, Vicente AC, Ostuki K, et al. Genetic variation and susceptibilities to protease inhibitors among subtype $\mathrm{B}$ and $\mathrm{F}$ isolates in Brazil. Antimicrob Agents Chemother 1999;43:253-8.

13. Brazilian Network for the HIV-1 Isolation and Characterization. HIV-1 diversity in Brazil: genetic, biological and immunological characterization of HIV-1 strains in three potential HIV vaccine evaluation sites. J Acquir Immune Defic Syndr 2000;23:184-93.

14. Campodonico M, Janssens W, Heyndrickx L, et al. HIV type 1 subtypes in Argentina and genetic heterogeneity of the V3 region. AIDS Res Hum Retroviruses 1996;12:79-81.

15. Marquina $\mathrm{S}$, Leitner TH, Rabinovich RD, et al. Coexistence of subtypes B, F, and B/F env recombinant of HIV type 1 in Buenos Aires, Argentina. AIDS Res Hum Retroviruses 1996;12:1651-4.

16. Arteinsten AW, Coppola J, Brown A, et al. Multiple introductions of HIV-1 subtype E into the western hemisphere. Lancet 1995; 346:1197-8.

17. Fernández RD, Jansson M, Halapi E, et al. Genetic analysis of V3 domain sequences obtained from Uruguayan HIV type 1-infected individuals. AIDS Res Hum Retroviruses 1996;12:1491-3.

18. Velarde-Dunois KG, Guimarães ML, La Fuente C, et al. Molecular characterization of human immunodeficiency virus type-1 infected individuals from Bolivia reveals the presence of two distinct genetic subtypes B and F. AIDS Res Hum Retroviruses 2000;16:1921-6.

19. Navas MC, Letourneur F, Gomas E, et al. Analysis of the V3 loop sequences from HIV type-1 infected patients from Colombia, South America. AIDS Res Hum Retroviruses 1999;15:1141-4.

20. Cabello A, Cabral M, Vera ME, et al. Analysis of V3 loop sequences from 10 HIV type 1-infected AIDS patients from Paraguay. AIDS Res Hum Retroviruses 1995;11:1135-7.

21. Lara C, Sallberg M, Johansson B, et al. The Honduran human immunodeficiency virus type 1 (HIV-1) epidemic is dominated by HIV-1 subtype B as determined by V3 domain sero- and genotyping. J Clin Microbiol 1997;35:783-4.

22. Rivera L, Rodríguez C, Novitsky V, et al. HIV-1 subtype B is a predominant virus of AIDS epidemic in Mexico [abstract 19]. Presented at the International Symposium on HIV, Leukemia and Opportunistic Cancers, Marrakech, May 23-28, 1999.

23. Desgranges C, Fillon S, Letourneur F, et al. HIV-1 subtypes in Santiago, Chile. AIDS 1998;12:1563-5.
24. Quiñones-Mateu ME, Dopazo J, Esté JA, et al. Molecular characterization of human immunodeficiency virus type 1 isolates from Venezuela. AIDS Res Hum Retroviruses 1995;11:605-16.

25. Quiñones-Mateu ME, Esteban D. Nucleotide diversity in three different genomic regions of Venezuelan HIV type 1 isolates: a subtyping update. AIDS Res Hum Retroviruses 1999;15:73-9.

26. Delgado E, León-Ponte M, Villahermosa ML, et al. Analysis of HIV type 1 protease and reverse transcriptase sequences from Venezuela for drug resistance-associated mutations and subtype classification: a UNAIDS study. AIDS Res Hum Retroviruses 2001;17:735-58.

27. Centro de investigaciones Económicas y Sociales, ONUSIDA, PNUD \& UNICEF. El VIH-SIDA en Venezuela: análisis de situación y recomendaciones. Caracas: 1998:1-149.

28. Delwart E, Shpaer EG, McCutchan FE, et al. Genetic relationships determined by a DNA heteroduplex mobility assay. Science 1993 ; 262:1257-61.

29. Bachmann MH, Deltwart E, Shpaer EG, et al. and WHO Network for HIV Isolation and Characterization. Rapid genetic characterization of HIV type 1 strains from four World Health Organizationsponsored vaccine evaluation sites using a heteroduplex mobility assay. AIDS Res Hum Retroviruses 1994;10:1345-53.

30. Zazzi M, Romano L, Brasini A, et al. Simultaneous amplification of multiple HIV-1 DNA Seqs from clinical specimens by using nested-primer polymerase chain reaction. AIDS Res Hum Retroviruses 1993;9:315-20.

31. Delwart EL, Herring B, Learn GH Jr, et al. Heteroduplex mobility analysis HIV-1 env subtyping kit, protocol version 3. Rockville, MD: NIH AIDS Research and Reference Program, 1995.

32. Brindeiro R, Vanderborght B, Caride E, et al. Sequence diversity of the reverse transcriptase of human immunodeficiency virus type 1 from untreated Brazilian individuals. Antimicrob Agents Chemother 1999;43:1674-80.

33. Thompson JD, Higgins D, Gibson T. CLUSTAL W-improving the sensitivity of progressive multiple sequence alignment through sequence weighting, position-specific gap penalities and weight matrix choice. Nucleic Acids Res 1994;22:4673-80.

34. Page RD. TreeView: an application to display phylogenetic trees on personal computers. Comput Appl Biosci 1996;12:357-8.

35. Kumar S, Tamura K, Jakobsen IB, et al. Molecular evolutionary genetics analysis, The Pennsylvania State University, PA: 2001. MEGA version 2.0.

36. Carr JK, Avila M, Carrillo MG, et al. Diverse BF recombinants have spread widely since the introduction of HIV-1 into South America. AIDS 2001;15:F41-7.

37. Bandea CL, Ramos A, Pieniazek D, et al. Epidemiological and evolutionary relationship between Romanian and Brazilian HIV-1 subtypes F strains. Emerg Infect Dis 1995;1:91-3.

38. Vicente ACP, Otsuki K, Silva NB, et al. The HIV epidemic in the Amazon basin is driven by prototypic and recombinant HIV-1 subtypes B and F. J Acquir Immune Defic Syndr 2000;23:327-31.

39. Thomson MM, Villahermosa ML, Vazquez E, et al. A B/F intersubtype-recombinant HIV-1 strain with recombination in Pol is widely circulating in Buenos Aires, Argentina [abstract 176]. Presented at the 7th Retrovirus Conference, San Francisco, January 2000.

40. Triques K, Bourgeois A, Saragosti S, et al. High diversity of HIV-1 subtype F strains in Central Africa. Virology 1999;259:99-109.

41. Janini LM, Pieniazek NJ, Peralta JM, et al. Identification of single and dual infections with distinct subtypes of human immunodeficiency virus type 1 by using restriction fragment length polymorphism analysis. Virus Genes 1996;13:69-81.

42. Heyndrickx L, Janssens W, Zekeng L, et al. and Study Group on Heterogeneity of HIV Epidemics in African Cities. Simplified strategy for detection of recombinant human immunodeficiency virus type 1 group $\mathrm{M}$ isolates by gag/env heteroduplex mobility assay. J Virol 2000;74:363-70

43. Descamps D, Calvez V, Izopet J, et al. and ANRS Antiretroviral Resistance Study Group. Prevalence of resistance mutations in antiretroviral-naive chronically HIV-infected patients in 1998: a French nationwide study. AIDS 2001;15:1777-82. 